Published as:

Ozturk Y., Bizzego A., Esposito G., Furlanello C., Venuti P.

Physiological and Self-report Responses of Parents of Children with Autism Spectrum Disorder to Children Crying.

Research in Developmental Disabilities, 73, 31-39.

https://www.sciencedirect.com/science/article/pii/S0891422217303116

\title{
Physiological and self-report responses of parents of children with autism spectrum disorder to children crying
}

Yagmur Ozturk $^{a}$, Andrea Bizzego ${ }^{b, c, d}$, Gianluca Esposito ${ }^{e, f}$, Cesare Furlanello ${ }^{b}$, Paola Venuti ${ }^{9}$,*

a Department of Brain and Behavioral Science, Psychology Section, University of Pavia, Pavia, Italy

b FBK - Fondazione Bruno Kessler, Trento, Italy

C Department of Information Engineering and Computer Science, University of Trento, Povo, Italy

d SKIL Telecom Italia, Trento, Italy

e Affiliative Behavior and Physiology Lab, Department of Psychology and Cognitive Sciences, University of Trento, Rovereto, Italy

f Social and Affiliative Neuroscience Lab, Division of Psychology, Nanyang Technological University, Singapore

9 Observation, Diagnosis and Education, Lab, Department of Psychology and Cognitive Science, University of Trento, Rovereto, Italy 


\section{Abstract}

Little is known about the physiological response of parents of children with Autism Spectrum Disorder (ASD) to crying of children who have already received the diagnosis of ASD. This study aimed to compare cardiac dynamics via Inter-Beat Interval (IBI) and self-reported emotional states of parents of children with ASD and of parents with typically developing (TD) children while listening to crying of children with ASD (ASD cry) and of typically developing children (TD cry). Analyses revealed higher $|B|$ in parents of children with $A S D$ than $|B|$ in parents of TD children while listening to both cry groups; however no differences on self-reported emotional states were observed. Parents of children with ASD were calmer (higher IBI) than parents of TD children while listening to crying. However, ASD cry did not elicit different IBI compared to TD cry. ASD cry and TD cry were differentiated based on parents' self-responses about what they felt during the listening of crying, their physiological responses showed no differences. These results highlight the similarities and differences between self-reported emotional states and physiological responses of parents of children with ASD, and also point to the importance of monitoring parents' physiological responses in addition to their subjective responses.

\section{Keywords}

- Parents of children with ASD;

- Physiological response;

- Self-reported emotional states;

- ASD cry 


\section{Introduction}

\subsection{Crying in typical and atypical development}

Autism Spectrum Disorder (ASD) is characterized by difficulty in social communication and restricted, repetitive patterns of behaviour and interests (American Psychiatric Association, 2013). The signs and symptoms of ASD vary widely, especially the child's social communication development which is important for spotting early signs of ASD. Recently, there has been an increasing interest in vocal development of children with ASD and typically developing (TD) children, in particular crying (Esposito, Nakazawa, Venuti, \& Bornstein, 2013; Esposito, Venuti, \& Bornstein, 2011; Esposito \& Venuti, 2009b, 2010b; Fort \& Manfredi, 1998; LaGasse, Neal, \& Lester, 2005; Manfredi, Bocchi, Orlandi, Spaccaterra, \& Donzelli, 2009; Oller et al., 2010; Orlandi, Manfredi, Bocchi, \& Scattoni, 2012; Reyes-Galaviz, Cano-Ortiz, \& Reyes-García, 2008; Sheinkopf, Iverson, Rinaldi, \& Lester, 2012; Venuti et al., 2012). Several studies reported that children with ASD express atypical patterns of distress vocalization such as higher fundamental frequency (f0), shorter inter-bout pauses, fewer utterances (Esposito, Nakazawa, Venuti, \& Bornstein, 2012; Esposito et al., 2011; Esposito, Nakazawa, et al., 2013; Esposito \& Venuti, 2009a, 2010a; Oller et al., 2010; Sheinkopf et al., 2012). Mostly, the literature in this field focused on crying of infants (such as 1-year old or new-borns). A recent study examined the acoustic characteristics of cries of 6-month-old infants at high risk of ASD (siblings of children with ASD) and found that they produced cries with higher and more variable f0 than infants not at risk for ASD (Sheinkopf et al., 2012). In addition to atypical patterns of distress vocalization, a number of studies focused on how listeners observe this cry type and showed that adults perceive cries of infants with atypical development differently compared to those of typically developing children (Esposito \& Venuti, 2008, 2009a, 2010; Venuti et al., 2012). 
Crying is one of the first communication channels utilized by infants to express their needs (Esposito \& Venuti, 2009b) and motivate the listener to respond (Zeskind \& Lester, 2001). From the caregivers' perspective, it is an important cue to understand what the infant wants and needs. They are alerted to attend to the cries and act to help alleviate the infant's distress through nursing and caring (Lin \& McFatter, 2012). It seems that crying regulates the child-caregiver relationship. An important question now arises of how caregivers of children with ASD feel when listening to the cries of children? Adults reported that they felt mainly uneasiness and negative states while listening to crying episodes belonging to children with $A S D$; more positive mental states were felt during the listening of crying episodes belonging to TD children (Esposito \& Venuti, 2008). Consequently, it is possible that parents fail to recognize the child's need and give adequate feedback. In line with this idea, a previous study investigated qualitatively different maternal reactions to the crying of infants later diagnosed with ASD compared to cries of typically developing infants and infants with developmental delays (Esposito \& Venuti, 2009a). The authors suggested that parents might find difficult to understand the meaning of crying episodes and might not know how to react (Esposito \& Venuti, 2009a). Similar results were found in a fMRI study in adults; Venuti et al. (2012) reported that cries of children with ASD, compared to those of typically developing children, elicited increased activity in brain regions associated with emotional processing, suggesting that ASD cries may elicit more negative feelings and may be perceived as more arousing compared to cries of TD children. In addition, recently, acoustic features have been highlighted as biomarkers and modulators of the expression of distress in ASD (Esposito, Hiroi, \& Scattoni, 2017). This expanded previous findings about the higher fundamental frequency (Esposito, Nakazawa, et al., 2013; Oller et al., 2010; Sheinkopf, Mundy, Oller, \& Steffens, 2000) as an acoustic characteristic of cries of children with ASD being accounted for the mental state uneasiness in listeners (Esposito et al., 2017; Esposito \& Venuti, 2010b). 
The research to date focused mostly on emotional responses of parents, i.e. how they felt while listening to crying, regardless of parental exposure. However, very little is known about physiological responses, and how they are modulated in parents of children with ASD. Recently, a study has pointed out similarities and differences between parents and non-parents' physiological responsiveness to cries of children with ASD (Esposito, Valenzi, Islam, \& Bornstein, 2015). In particular, fathers showed higher Inter-Beat Interval (IBI) than non-fathers while listening to typical and atypical cries, indicating that fathers were calmer than non-fathers while listening to cries, and the authors suggested that perhaps because fathers have more exposure to or experience in caring for crying. Moreover, previous studies examined the responses to infant crying; little is known about the responses of parents of children with ASD to crying of children who have already received the diagnosis of ASD.

\subsection{Current study}

This study therefore set out to explore both physiological responses and self-reported emotional states of parents of children with ASD when listening to crying of children diagnosed with ASD. Regarding self-reports we aimed to discover the dimensional nature of self-reported emotional responses such as valence, arousal and stress. Assumed that self-reports of a current emotion are likely to be more valid than self-reports concerning past, future, or trait-related experiences of emotion (Mauss \& Robinson, 2009; Robinson \& Clore, 2002), we particularly chose self-reports of current emotional responses of parents about cry episodes belonging to either children with ASD or TD children. Moreover, we employed a physiological evaluation of calm versus stress by monitoring cardiac activity: cardiac Inter-Beat Interval (IBI; the inverse of heart rate), which is a temporal distance in millisecond between two consecutive heartbeats, was considered, because increases of IBI usually happen in a calming condition, while IBI decrease is often associated with 
stress (Esposito et al., 2015). The IBI was used to be an indicator in the literature, considering several fields such as emotional concordance (Butler, Gross, \& Barnard, 2014), empathy (Oliveira-Silva \& Gonçalves, 2011), emotion regulation (Hannesdóttir, Doxie, Ann Bell, Ollendick, \& Wolfe, 2010) and infant calming responses (Esposito, Yoshida, et al., 2013). Moreover, it is more reliable than other indicators (for instance: frequency domain indicators) as it could be more accurately estimated from short segments (McNames \& Aboy, 2006).

Our study attempts to answer two main questions: 1) Do parents of children with ASD and parents with TD children report different levels of stress, arousal and negative valence and show different physiological responses (Inter-beat Interval - IBI) when listening to cry episodes belonging to children with ASD? 2) Do parents report more stress, arousal and negative valence for cry episodes belonging to children with ASD? Do cry episodes of children with ASD elicit lower IBI, compared to cry episodes of TD children?

Guided by an extensive literature showing that cry episodes belonging to children with ASD are perceived as more aversive and elicit more negative feelings compared to cry episodes of TD children, we developed two main hypotheses. We expected that parents of children with ASD can discriminate cry episodes belonging to children with ASD cry from cry episodes of TD children, based on higher stress, arousal and negative valence. Second, we expected that parents of children with ASD will keep IBI higher than parents of TD children while listening to ASD cry, because of their experience with facing the many challenging situations (including crying episodes) caused by the condition (Rivard, Terroux, Parent-Boursier, \& Mercier, 2014). This is also in line with a previous study in which fathers were found to be calmer than non-fathers while listening to cries (Esposito et al., 2015). 
It is worth to note that the present study tested the hypothesis focusing on the emotional and physiological responses rather than on a direct evaluation (such as by asking parents to judge whether a cry was from an ASD child or a TD child). The information about the presence of possible atypical sounds was hidden. A direct question might indeed create a bias or a specific rise of attention; indeed the self-rating phase was postponed and does not include reference to ASD.

\section{Methods}

\subsection{Participants}

The participants comprised of 30 parents (15 mothers and 15 fathers) of typically developing (TD) children and 19 parents (11 mothers and 8 fathers) of children diagnosed with ASD. Parents of TD children were aged 36 years $(S D=6.7)$ and parents of children with $A S D$ were aged 40 years $(S D=3.3)$. The mean age of mothers of children with ASD was 40.5 years $(S D=3.6)$ and fathers' mean age was 40.6 years $(S D=3.0)$, whereas the mean age of mothers of TD children was 33.9 years $(S D=5.9)$ and fathers' mean age was 38.1 years $(S D=7.0)$. Parents of children with $A S D$ were consecutive families referred to the XY lab purposely left blank of the University of XY purposely left blank, and accepting to participate. Parents of typically developing children were recruited through an announcement; parents with children matching age of the atypical sample were considered. All parents' ethnic group was Italian, and they were all married. The diagnosis of children was confirmed through clinical judgement by an independent clinician based on the DSM-IV-TR criteria for Pervasive Developmental Disorders (PDD) as well as through the Autism Diagnostic Observation Schedule-Generic (ADOS-G; Lord et al., 2000) in the the XY lab purposely left blank of the University of XY purposely left blank. All of them 
were classified as Autistic (F84.0). It is worth to note that the video recordings were collected during the diagnosis process before DSM-V and ADOS-2 were published in Italy. Parents did not show clinical levels of stress $(M=62.5, S D=17.2)$; based on the cut-off for the Parenting Stress Index-Short Form Total Stress Score (Abidin, 1995). Parent did not show also high levels of depression and anxiety (Depression: $M=3.5, S D=3.2$; Anxiety: $M=3.7, S D=3.3$ ), considering the psychometric properties of the Depression, Anxiety Stress Scales-21 (DASS-21; Lovibond \& Lovibond, 1995) on clinical sample (patients whose most severe problem was either diagnosed depressive disorders or any diagnosed anxiety disorder) who reside in Italy (Bottesi et al., 2015). The socioeconomic status (SES) of the parents was calculated using the Four-Factor Index of Social Status (Hollingshead, 1975; Rossi, 1994). Our sample comprised of mainly middle class Italians. SES of parents of typically developing children $(M=43.7, S D=13.2, M d n=40.5)$ and parents of children diagnosed with $A S D(M=41.8, S D=13.2, M d n=40.5)$ did not differ significantly $(W=$ $1781.5, p>.05)$

Table 1.

Demographic characteristics of participating 49 parents.

\begin{tabular}{lll} 
& \multicolumn{2}{l}{ Group } \\
\cline { 2 - 3 } & Parents of children with ASD & Parents of TD children \\
& $\mathrm{M}(\mathrm{SD})$ & $\mathrm{M}(\mathrm{SD})$ \\
\hline Age (year) & $40(3.3)$ & $36(6.7)$ \\
\hline SES & $37.2(11.9)$ & $44.1(15.1)$ \\
\hline PSI - Total stress & $65.5(28.3)$ & $60.7(26.4)$ \\
\hline DASS- Depression & $3.3(3.2)$ & $3.8(2.9)$ \\
\hline DASS- Anxiety & $3.3(3.0)$ & $4.9(4.0)$ \\
\hline WNSS & $3.6(0.8)$ & $3.5(0.6)$ \\
\hline
\end{tabular}

Note. SES: Socioeconomic status; PSI: Parenting Stress Index; DASS: Depression Anxiety Stress Scale; WNSS: Weinstein Noise Sensitivity Scale; M: Mean; SD: Standard Deviation. 


\subsection{Acoustic Stimuli}

Two groups of cry episodes were used in the present study. These cry episodes belong to either children later diagnosed with ASD (throughout this paper, referred to as an ASD cry) or aged-matched typically developing children (referred to as a TD cry). The range of age was $36-52$ months. ASD cries were extracted from video recordings, which were collected during the diagnosis process in the XY lab purposely left blank; whereas cries of typically developing children were recorded in the XY lab purposely left blank specifically for the present study. The range of age was $36-52$ months. Parents ensured that children were not hungry or tired and did not have any specific pain during the video recordings. The diagnosis was confirmed based on the DSM-IV-R criteria, confirmed by the Autism Diagnostic Observation Schedule, a standardized diagnostic observational instrument that quantifies ASD symptoms in social reciprocity, communication, play and repetitive behaviors (ADOS-G; Lord et al., 2000). Additionally, considering ethical aspects, we underline that all crying episodes were produced without explicit elicitation. Acoustic data were extracted as segments of records a structured diagnostic assessment, and indeed crying episodes are not frequent.

Each cry episode lasts 6 seconds. In total, 15 cry episodes were used in the present study: 8 ASD cry episodes and 7 TD cry episodes. It is worth to note that they were selected from 15 different children who are not sons/daughters of the participants.

The cry episodes were extracted from the video employing the open source Audacity software (version 2.0.2) for audio editing to produce separate audio files for the groups. Then they were digitized and analyzed using Praat, an acoustic analysis software (Boersma \& Weenink, 2001). We obtained the fundamental frequency (f0) of each cry episode to obtain the acoustic features. A long-term average spectrum (LTAS) was employed to provide spectral information for each cry episode. LTAS is used to 
discriminate cry characteristics of different categories of children (Lin \& Green, 2007). Each cry episode was segmented in $1000 \mathrm{~ms}$ windows. Frequencies outside the $200 \mathrm{~Hz}$ to $1200 \mathrm{~Hz}$ were filtered out to remove frequencies that are outside of the cry range and due to noise (Abou-Abbas, Tadj, \& Fersaie, 2017; Esposito et al., 2015, 2011; Sheinkopf et al., 2012). The First Spectral Peak (FSP) was obtained by identifying the value of the first amplitude peak of the LTAS output. FSP (in $\mathrm{Hz}$ ) is an estimate of the average f0 of the episode of crying (Lin \& Green, 2007). The FSPs of cry episodes were significantly different between ASD $(M=514.07, S D=7.04)$ and TD $(M=492.73, S D=6.93)$ cry, with $t(13)=5.90, p<.001$. Furthermore, all acoustic files were edited to standardize them for volume and duration (6 seconds).

\subsection{Procedure: Stimuli presentation and response measurements}

The experiment was structured into two parts, for physiological and behavioral data acquisition separately. Each participant completed a 10 seconds baseline session at the beginning of the experiment in which he/she was asked to remain still to avoid any motion artifact in physiological signals.

The first part contains 32 sessions: 16 sessions of ASD cry and 16 sessions of TD cry. Each session consists of two phases: sound (a cry episode which lasts 6s) and rest (10s). It is worth to note that the experimental design was based on literature on physiological responses to emotional stimulation by affective auditory and visual stimuli (Anttonen \& Surakka, 2005; Bradley \& Lang, 2000; Bradley, Miccoli, Escrig, \& Lang, 2008; Lang, Greenwald, Bradley, \& Hamm, 1993; Madan, Harrison, \& Mathewson, 2017; Verona, Patrick, Curtin, Bradley, \& Lang, 2004; Zhou, Qu, Jiao, \& Helander, 2014). The order of the cry episodes was randomized for each participant. A graphical representation of a session is presented in Fig 1. Participants were asked to listen to each sound presented by means a personal computer speaker and then to rest without moving. They were not 
informed about the aim of the study; in particular, they did not know that some of the sounds were ASD cries. Only during the first part of the experiment, the Blood Volume Pulse (BVP) signal was recorded with an Empatica E4 device, a wearable wireless multisensor wristband (Garbarino, Lai, Bender, Picard, \& Tognetti, 2014). Non-dominant arm was used to place the device and all subjects indicated their left arms as nondominant. To reach a total of 32 sessions, each cry was repeated twice in order to have enough samples for the physiological analysis; with two cry episodes from the TD cry group repeated three times (they were chosen by the experiment software randomly).

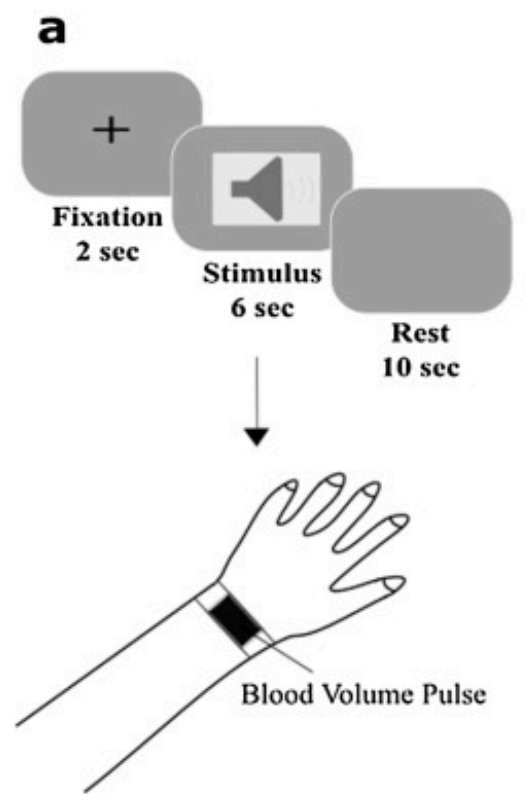

\section{b}

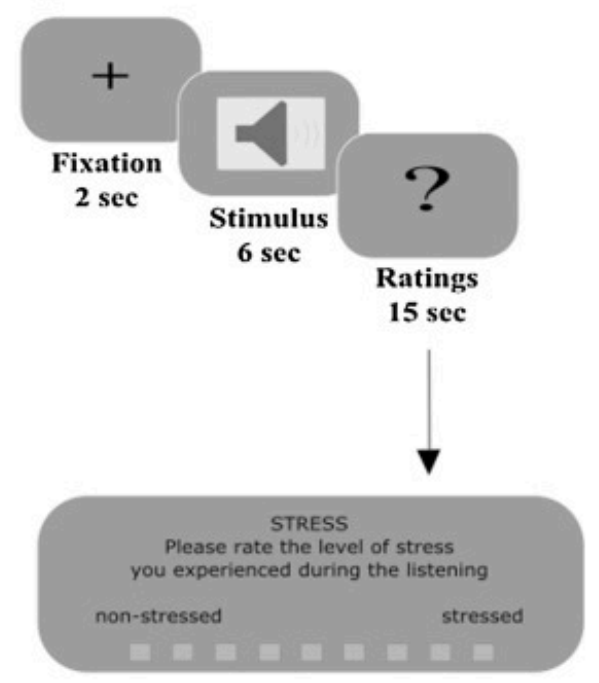

Fig. 1. The graphical representation of the structure of each session and measures. (a) The physiological data acquisition sessions. Measures of the blood volume pulse were collected using a wearable wristband device while subjects were listening to the stimuli. (b) The self-report data acquisition sessions. Subjective emotional ratings on stress, arousal and negative valence were collected for each stimulus.

The second part contains 15 sessions; each session consists of two phases: sound (6s) and rating (15s: 3 scales $\times 5$ seconds). Each cry was repeated once, thus participants rated 15 crying episodes. Participants were asked to listen to each sound then rate that 
sound using 9-point Likert-scales (see Fig 1). There were 3 rating scales to measure subjects' emotional responses to the stimuli: stress, arousal and negative valence. The valence and arousal rating scales of the Self-Assessment Manikin (SAM; Bradley \& Lang, 1994) were used to measure valence and arousal. Regarding the stress scale, we asked to subjects to rate their level of stress they experience during the listening. Higher scores indicate high level of stress and arousal, and less negative valence (higher valence). It is worth to note that each cry was rated using three scales only one time.

After the first part, subjects were allowed to have a break and then continue the experiment when they feel comfortable. The wristband was removed from the arm of the subject after the first part ended in order to make the subjects feel more comfortable during the rating phase. Experiment lasted approximately half an hour. Participants were familiarized with the rating procedure during the training session before the experimental session. Volume of the device was kept at the same level for all subjects.

It is worth to note that there are three reasons for having two separate parts: (1) The selfrating procedure might cause effect on the physiological responses while the participants were thinking about their feeling; (2) The self-rating procedure might cause motion artifacts in physiological signals due to using a computer mouse to rate; (3) In order to have more repetitions to improve the statistical power, the physiological data acquisition section was designed as it consists of 32 sessions (sounds with their repetitions), whereas participants listened and rated the cry episodes only once in behavioral data acquisition.

Furthermore, participants completed three questionnaires the day before the experiments to examine whether there was an association between parents' responses to crying (outcome variables) and their depression, anxiety, parenting stress or noise sensitivity. The three questionnaires are the Parenting Stress Index-Short Form (PSI/SF; Abidin, 1995), the Depression, Anxiety Stress Scales-21 (DASS-21; Lovibond \& Lovibond, 1995) 
and Weinstein Noise Sensitivity Scale (WNSS; Senese, Ruotolo, Ruggiero, \& lachini, 2012).

\subsection{Data Analysis}

Regarding behavioral data analysis, the values from participants' self-reports for the sounds within the same cry category (i.e., the values for 8 ASD cry episodes within ASD cry category) were averaged to create a score for each category (i.e., "ASD cry" score). Then, we performed Wilcoxon tests (the assumption for normality has not been met), and reported the results considering the Bonferroni multiple comparison correction. We estimated effect sizes from the $p$-values reported using the formula: $r=Z / \sqrt{ } N$ (Rosenthal, 1991).

Regarding the physiological data analysis, all sounds, including repetitions, were considered. We focused on computing the mean Inter-beat Interval (IBI) length over a subset of 31 subjects (20 parents of TD children and 11 parents of children with ASD), having dropped sessions incomplete due to equipment malfunctioning. The IBI series was extracted from the Blood Volume Pulse (BVP) signal acquired using the derivative based detection algorithm (Bizzego \& Furlanello, 2017). The mean IBI was then computed for each stimulus ( 6 seconds) of the first part of the experiment, thus obtaining 32 data points for each subject (the first part contains 32 sessions: 16 sessions of ASD cry and 16 sessions of TD cry). In addition, we also computed the mean IBI value during the baseline session (10 seconds). To account for the inter-subject variability, the mean IBI values were normalized by subtracting the mean IBI baseline. The computations were made using the pyHRV Python library (Bizzego, Mina, Zarbo, Esposito, \& Furlanello, 2014). Finally we performed non-parametric tests (namely Wilcoxon tests), because the normality assumption has not been met. The results with Median $(M d n)$, test statistic $(W)$, p-value $(p)$, effect size (ES) were reported. The effect sizes were calculated in the same way as for 
the behavioral data analysis. All statistical analyses were performed using the $R$ computing environment (R Core Team, 2014).

For both parts, the associations between the output scores/ratings and the following variables were examined: parents' age, family SES, the total score of the PSI/SF and the score from the WNSS.

\section{Results}

No associations emerged between the output and parents' age, family SES, the total score from the PSI, the subscale scores from the DASS or the score from the WNSS. The baseline IBI was not significantly different between Parents of children with ASD and Parents of TD children $(W=94, p=.53)$. Stimulus order had only a small effect on stress rating (TD parents: $\rho=0.10, p=.03$; ASD parents: $\rho=0.12, p=0.05$ ), and no significant relationship with the other ratings, suggesting that effects of habituation were kept to an acceptable minimum. To consider data dependence, we performed Kendall rank correlation analyses, finding that scores of female and male subjects of married couples were not correlated (all ps > .05).

The rest of the result section is divided into two parts. The first part deals with comparisons between the responses of Parents of children with ASD and Parents of TD children, in order to answer the first research question of this study. The second part focuses on the comparisons between the participants' responses to two cry categories (ASD cry and TD cry). Additionally, we reported the results that emerged from the analysis of the gender differences (female versus male) in only Inter-beat Interval (due to the small sample size for the self-reported data, we did not perform this analysis for stress, arousal and valence).

3.1. Comparisons between Parents of children with ASD and Parents of TD children 


\subsubsection{Stress}

There is no difference between Parents of children with ASD $(M d n=6.4)$ and Parents of TD children $(M d n=5.8)$ in their self-reported stress levels for ASD cry; $W=310.5, p=.61$, $\mathrm{ES}=-0.07$. Similarly, there is no difference between Parents of children with ASD (Mdn $=$ 4.9) and Parents of TD children $(M d n=3.6)$ in their self-reported stress levels for TD cry; $W=373, p=.07, \mathrm{ES}=-0.26$.

\subsubsection{Arousal}

There is no difference between Parents of children with ASD $(M d n=6.4)$ and Parents of TD children $(M d n=5.3)$ in their self-reported arousal levels for ASD cry; $W=328.5, p=$ .38 , ES $=-0.13$. Moreover, there is no difference between Parents of children with ASD $(M d n=4.3)$ and Parents of TD children $(M d n=3.3)$ in their self-reported arousal levels for TD cry; $W=355, p=.15, \mathrm{ES}=-0.20$.

\subsubsection{Negative Valence}

As it is reported in the Method section, higher scores indicate lower negative valence (scale from 1 to 9 ). There is no difference between Parents of children with ASD (Mdn = 3.1) and Parents of TD children $(M d n=2.9)$ in their self-reported valence levels for ASD cry; $W=282.5, p=.97, E S=-0.01$. However, there is a statistical difference in selfreported valence levels for TD cry between Parents of children with ASD and Parents of TD children; $W=174, p=.02$, ES $=0.32$. Parents of TD children $(M d n=4.4)$ reported higher scores (meaning that lower valence) than Parents of children with ASD $(M d n=3.8)$. However, the results are not significant after Bonferroni correction.

\subsubsection{Inter-beat Interval (IBI)}


Regarding the comparisons between Parents of children with ASD and Parents of TD children, there are significant differences (Fig 2): Parents of children with ASD had higher IBI than Parents of TD children during both ASD cry $(W=30440, p=.003$, ES $=-0.21)$ and TD cry $(W=32522, p<.001, \mathrm{ES}=-0.28)$.
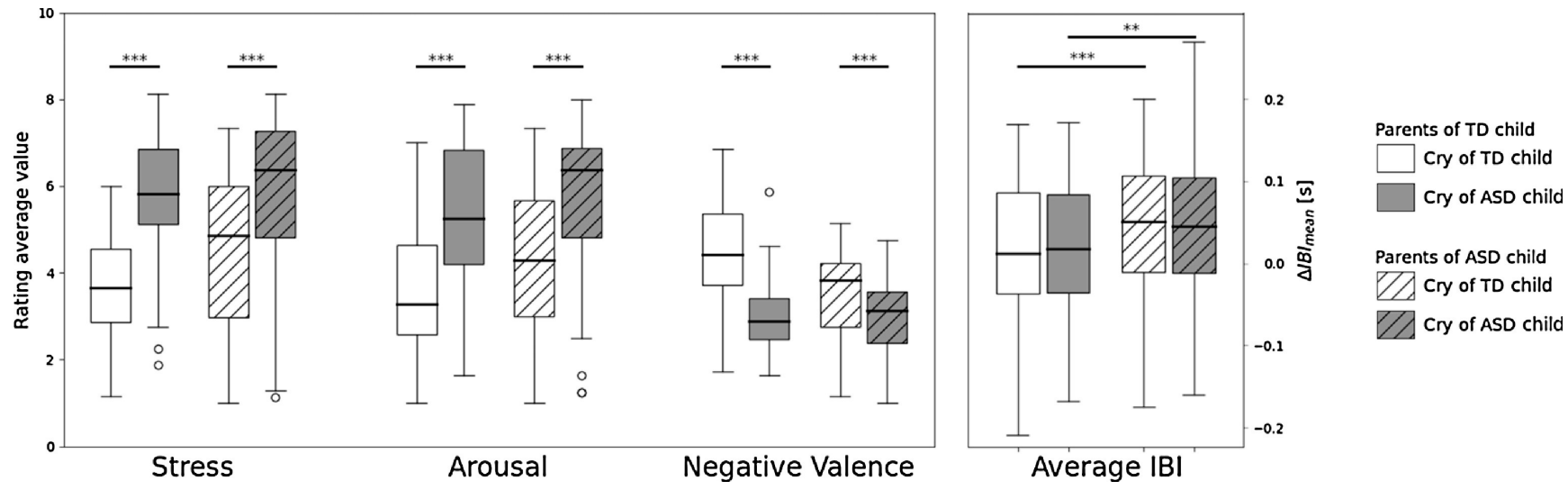

Fig. 2. Mean levels of ratings and Inter-Beat Interval of parents of typically developing children and parents of children with ASD while listening to the two types of cry stimuli (Typically developing children's cries and cries of children with ASD). Higher scores indicate high level of stress and arousal, and less negative valence. ${ }^{* * *} p<0.001$, ${ }^{* *} p<0.01,{ }^{*} p<0.05$.

\subsection{Comparisons between cry categories (ASD cry and TD cry)}

\subsubsection{Stress}

In parents of children with ASD, self-reported stress levels were significantly higher for ASD cry $(M d n=6.4)$ than for TD cry $(M d n=4.9) ; W=172, p<.001$, ES $=-0.54$. Similarly, parents of TD children reported more stress for the ASD cry $(M d n=5.8)$ compared to TD $\operatorname{cry}(M d n=3.6) ; W=465, p<.001, \mathrm{ES}=-0.78($ Fig 2$)$.

\subsubsection{Arousal}

There is a significant difference between self-reported arousal levels of Parents of children with ASD to ASD cry $(M d n=6.4)$ and to TD cry $(M d n=4.3) ; W=188, p<.001$, ES = - 
0.71. Also, Parents of TD children reported as more aroused for ASD cry $(M d n=5.3)$ compare to TD cry $(M d n=3.3) ; W=432, p<.001$, ES $=-0.60$ (Fig 2).

\subsubsection{Negative Valence}

There is a significant difference between self-reported valence levels of Parents of children with $\mathrm{ASD}$ that perceived the $\mathrm{ASD}$ cry $(M d n=3.1)$ more negatively than $\mathrm{TD}$ cry $(M d n=$ 3.8); $W=7, p<.001, E S=-0.64$. Similarly, parents of TD children reported lower valence for ASD cry $(M d n=2.9)$ compared to TD cry $(M d n=4.4) ; W=4, p<.001$, ES = -0.61 (Fig 2). It is worth to note that higher scores indicate lower negative valence.

\subsubsection{Inter-beat Interval (IBI)}

IBI does not differentiate during TD cry and during ASD cry, neither in Parents of TD children $(W=49279, p=.58, \mathrm{ES}=-0.03)$ nor Parents of children with ASD $(W=14062, p$ $=.54, \mathrm{ES}=-0.04)$.

\subsection{Comparisons between Mothers and Fathers}

Fathers of children with ASD showed higher $|B|$ than mothers during both ASD cry $(W=$ 4414, $p<.001, \mathrm{ES}=-0.39)$ and TD cry $(W=4495, p<.001, \mathrm{ES}=-0.40)$, but the difference was not observed for TD parents (ASD cry: $W=8552.5, p=.14$, ES =-0.08, TD cry: $W=8957.5, p=0.27, E S=-0.06)$.

\section{Discussion}

The present study set out to explore the responses of parents of children with ASD, in particular their physiological activity and their emotional self-reports when listening to ASD 
child crying. Returning to the research questions posed at the beginning of this study, it is now possible to state that: 1) Higher IBI in parents of children with ASD compared to parents of TD children was observed during listening of crying of children (either with ASD or TD); however, no difference on self-reported emotional states emerged. 2) ASD cry did not elicit different IBI compared to TD cry, although both parent types reported more stress, arousal and negative valence for the ASD cry.

Regarding the comparison between the responses of two types of parents, self-reported stress and arousal levels of parents of children with ASD and parents of TD children were not different; however, their physiological responses presented a different pattern. Parents of children with ASD showed higher IBI compared to parents of TD children. Specifically, parents of children with ASD show more physiological reactivity (with higher IBI reflecting a calming) than parents of TD children while listening to crying.

This result seem to confirm that parents, who live with a child with ASD and have more exposure to caring for crying children with ASD can physiologically cope slightly better. Eventually, they have their own strategy to provide care, manage their child's behavior and deal with difficulties. Indeed, an explorative study on parenting behaviors among parents of children with ASD highlighted that they use more specific parenting behaviors; they more explicitly stimulate their child's development (Lambrechts, Van Leeuwen, Boonen, Maes, \& Noens, 2011). It is expected that they have more responsibilities, such as accommodating some behaviors that are the characteristics of ASD. Parents of children with ASD are expected to play the roles of both nurturer and trainer so that they can meet the nurturing and behavioral training needs of their child with ASD by themselves (Tsai, Tsai, \& Lotus Shyu, 2008). In line with this, a recent qualitative study showed that although parents of children with ASD need to play multiple roles, focus on their child's needs and find a balance, they are determined to strive for balance within the family (Hoogsteen \& 
Woodgate, 2013). As crying can be considered one of many challenging situations parents experience in their daily life, it is possible that parents with a child with ASD have their own strategy to deal with crying, such as staying calmer (with higher IBI compared to that of parents of TD children in our study) to crying. This is also in line with the previous study in which adults with parenting experiences (fathers) were found to be calmer (higher IBI) than non-parents while listening to cries (Esposito et al., 2015). Interestingly, these differences and similarities were observed not only during the listening of ASD cry, but also TD cry. Both cry types elicit higher IBI parents of children with ASD, compare to parents of TD children.

With respect to the second research question, we found that an ASD cry was reported as more stressful, arousing and elicits more negative valence, compared to a TD cry, by both parents of children with ASD and parents of TD children. This is an expected result, as children with ASD express atypical patterns of distress vocalization, such as higher f0 (Esposito et al., 2012, 2011; Esposito, Nakazawa, et al., 2013; Esposito \& Venuti, 2009a, 2010a; Oller et al., 2010; Sheinkopf et al., 2012). Moreover, these results are in line with those of previous studies which indicate that adults felt mainly uneasiness and negative states while listening to ASD crying episodes and felt more positive mental states while listening to crying episodes of TD children (Esposito \& Venuti, 2008, 2009a, 2009b, 2010; Venuti et al., 2012).

It is somewhat surprising that no differences were found in parents' physiological responses (their Inter-Beat Interval - IBI) for crying type. Once again, there are two different patterns between emotional self-reports and physiological responses of parents. Even though ASD cry and TD cry were differentiated based on participants' self-responses about what they felt during the listening of crying, their physiological responses showed no differences. A possible explanation for this may be the same fact we mentioned above, 
parents of children with ASD have experience in caring for children with ASD cry (Esposito \& Venuti, 2008). It may be that physiological responses are no more differentiated between the listening of $\mathrm{ASD}$ and TD cries; both cry types trigger their physiological structures similarly so that they have a similar Inter-beat Interval for the two cry types. Also, it is important for parent-child relationship, since crying represents a signal through which parents shape their own caregiving. More research on the physiological responses of parents is therefore needed. A further study could test physiological responses of parents measuring other components such as hand temperature, as an increase in skin temperature of the right hand is associated with promptness to action (Esposito et al., 2015; Rimm-Kaufman \& Kagan, 1996).

This study has a number of strengths, including the focus on parents of children with ASD and measure of both parents' emotional and physiological mechanisms. Moreover, our findings have potential implications also for early identification of ASD; specific questions regarding children cry and overall attention to this issue could be developed for nurses and paediatrician during regular screening visit also before 2 years. A more immediate application of this work regards parent support, because indicators derived from physiological responses may help explaining to parents how to distinguish between a usual parental feeling and specific negative valence or stronger arousal motivated by atypical patterns. However, some relevant limitations must be noted. We analyzed relatively small samples (30 parents of typically developing children and 19 parents of children diagnosed with ASD); larger samples and also parents of children with other developmental disabilities may be informative. Moreover, the study had missing data on IBI. The adoption of wearable devices for HR in this research framework opens interesting research and applicative opportunities; however the lower robustness with respect to medical grade devices introduces limitations such as missing data. Aspects about 
robustness and reproducibility of wearable devices are discussed in Bizzego (2017). Lastly, the lack of labelling the acoustic stimuli by pain, hunger or tiredness cry types should be considered as a limitation.

This research may throw up many questions in need of further investigation, such as a research may be done to determine the connection with ASD heritability. It could be tested using the Broader Autism Phenotype Interview. Also, it would be interesting to investigate the role another feature which the difference in history of parents, as it requires a complete evaluation of personal history, for instance having raised other children before as an older siblings, or direct parental experience with previous children.

In conclusion, we believe that this study highlights the similarities and differences between self-reported emotional states and physiological responses of parents of children with ASD; cardiovascular responses of parents do not differ during the listening of ASD and TD cry (they showed similar IBI during the listening of ASD cry and TD cry), even though they reported more stress, arousal and negative valence for ASD cry. This study, through the focus on physiological aspects of parental response in parents of children with ASD, provides novel stepping-stones towards enhancing our understanding of caregiver responses to children crying. Moreover, this research has a number of practical applications; such as focusing on physiological responses of parents towards crying led us to have a better understanding of their reactions towards crying of children with ASD. It is important to pay attention to crying of children and parents' responses which can be observed also during the diagnosis of ASD.

\section{Acknowledgements}


All authors sincerely thank the families for their time and participation. The authors also

thank Daniela Ambrosi for her support during data collection. This research project was supported by the XY lab purposely left blank at the University of XY purposely left blank.

One of the authors was supported by a fellowship from TIM.

\section{References}

Abidin, R. R. (1995). Parenting Stress Index: Professional manual (3rd ed.). Odessa, FL: Psychological Assessment Resources.

Abou-Abbas, L., Tadj, C., \& Fersaie, H. A. (2017). A fully automated approach for baby cry signal segmentation and boundary detection of expiratory and inspiratory episodes. The Journal of the Acoustical Society of America, 142(3), 1318-1331.

American Psychiatric Association. (2013). The Diagnostic and Statistical Manual of Mental Disorders: DSM 5. Arlington,VA: APA.

Anttonen, J., \& Surakka, V. (2005). Emotions and heart rate while sitting on a chair. In Proceedings of the SIGCHI conference on Human factors in computing systems - $\mathrm{CHI}$ '05 (p. 491). New York, New York, USA: ACM Press.

Bizzego, A. (2017). A data analytics framework for physiological signals from wearable devices. University of Trento. Retrieved from http://eprints-phd.biblio.unitn.it/2089/

Bizzego, A., \& Furlanello, C. (2017). DBD-RCO: Derivative Based Detection And Reverse Combinatorial Optimization To Improve Heart Beat Detection For Wearable Devices. bioRxiv, 118943.

Bizzego, A., Mina, M., Zarbo, C., Esposito, G., \& Furlanello, C. (2014). Physiolyze: A Galaxy-based web service for Heart Rate Variability analysis with online processing.

Boersma, P., \& Weenink, D. (2001). Praat, a system for doing phonetics by computer. Glot International, 5(9/10), 341-45.

Bottesi, G., Ghisi, M., Altoè, G., Conforti, E., Melli, G., \& Sica, C. (2015). The Italian version of the Depression Anxiety Stress Scales-21: Factor structure and psychometric properties on community and clinical samples.

Bradley, M. M., \& Lang, P. J. (1994). Measuring emotion: the Self-Assessment Manikin and the Semantic Differential. Journal of Behavior Therapy and Experimental Psychiatry, 25(1), 49-59.

Bradley, M. M., \& Lang, P. J. (2000). Measuring emotion: Behavior, feeling, and physiology.

Bradley, M. M., Miccoli, L., Escrig, M. A., \& Lang, P. J. (2008). The pupil as a measure of emotional arousal and autonomic activation. Psychophysiology, 45(4), 602-607.

Butler, E. A., Gross, J. J., \& Barnard, K. (2014). Testing the effects of suppression and reappraisal on emotional concordance using a multivariate multilevel model. Biological Psychology, 98, 6-18.

Esposito, G., Hiroi, N., \& Scattoni, M. L. (2017). Cry, Baby, Cry: Expression of Distress As a Biomarker and Modulator in Autism Spectrum Disorder. International Journal of Neuropsychopharmacology, 20(6), 498-503. 
Esposito, G., Nakazawa, J., Venuti, P., \& Bornstein, M. H. (2012). Perceptions of distress in young children with autism compared to typically developing children: a cultural comparison between Japan and Italy. Research in Developmental Disabilities, 33(4), 1059-67.

Esposito, G., Nakazawa, J., Venuti, P., \& Bornstein, M. H. (2013). Componential deconstruction of infant distress vocalizations via tree-based models: a study of cry in autism spectrum disorder and typical development. Research in Developmental Disabilities, 34(9), 2717-24.

Esposito, G., Valenzi, S., Islam, T., \& Bornstein, M. H. (2015). Three physiological responses in fathers and non-fathers' to vocalizations of typically developing infants and infants with Autism Spectrum Disorder. Research in Developmental Disabilities, 43-44, 43-50.

Esposito, G., \& Venuti, P. (2008). How is crying perceived in children with Autistic Spectrum Disorder. Research in Autism Spectrum Disorders, 2(2), 371-84.

Esposito, G., \& Venuti, P. (2009a). Comparative Analysis of Crying in Children with Autism, Developmental Delays, and Typical Development. Focus on Autism and Other Developmental Disabilities, 24(4), 240-47.

Esposito, G., \& Venuti, P. (2009b). Typical and atypical expression of distress: a study on cry. International Public Health Journal, 1(2), 141-50.

Esposito, G., \& Venuti, P. (2010a). Developmental changes in the fundamental frequency (f0) of infants' cries: a study of children with Autism Spectrum Disorder. Early Child Development and Care, 180(8), 1093-102.

Esposito, G., \& Venuti, P. (2010b). Understanding early communication signals in autism: a study of the perception of infants' cry. Journal of Intellectual Disability Research : JIDR, 54(3), 216-23.

Esposito, G., Venuti, P., \& Bornstein, M. H. (2011). Assessment of distress in young children: a comparison of autistic disorder, developmental delay, and typical development. Research in Autism Spectrum Disorders, 5(4), 1510-16.

Esposito, G., Yoshida, S., Ohnishi, R., Tsuneoka, Y., del Carmen Rostagno, M., Yokota, S., ... Venuti. (2013). Infant Calming Responses during Maternal Carrying in Humans and Mice. Current Biology, 23(9), 739-745.

Fort, A., \& Manfredi, C. (1998). Acoustic analysis of newborn infant cry signals. Medical Engineering \& Physics, 20(6), 432-442.

Garbarino, M., Lai, M., Bender, D., Picard, R. W., \& Tognetti, S. (2014). Empatica E3 - A wearable wireless multi-sensor device for real-time computerized biofeedback and data acquisition. Wireless Mobile Communication and Healthcare (Mobihealth), 2014 EAl 4th International Conference on.

Hannesdóttir, D. K., Doxie, J., Ann Bell, M., Ollendick, T. H., \& Wolfe, C. D. (2010). A Longitudinal Study of Emotion Regulation and Anxiety in Middle Childhood: Associations with Frontal EEG Asymmetry in Early Childhood. Developmental Psychobiology, 52(2), 197-204.

Hollingshead, A. B. (1975). Four factor index of social status (Unpublished manuscript). New Haven, CT: Yale University.

Hoogsteen, L., \& Woodgate, R. L. (2013). Centering autism within the family: a qualitative approach to autism and the family. Journal of Pediatric Nursing, 28(2), 135-40. 
LaGasse, L. L., Neal, A. R., \& Lester, B. M. (2005). Assessment of infant cry: acoustic cry analysis and parental perception. Mental Retardation and Developmental Disabilities Research Reviews, 11(1), 83-93.

Lambrechts, G., Van Leeuwen, K., Boonen, H., Maes, B., \& Noens, I. (2011). Parenting behaviour among parents of children with autism spectrum disorder. Research in Autism Spectrum Disorders, 5(3), 1143-52.

Lang, P. J., Greenwald, M. K., Bradley, M. M., \& Hamm, A. O. (1993). Looking at pictures: affective, facial, visceral, and behavioral reactions. Psychophysiology, 30(3), 261-73.

Lin, H.-C., \& Green, J. A. (2007). Effects of Posture on Newborn Crying. Infancy, 11(2), 175-89.

Lin, H.-C., \& McFatter, R. (2012). Empathy and distress: two distinct but related emotions in response to infant crying. Infant Behavior \& Development, 35(4), 887-97.

Lord, C., Risi, S., Lambrecht, L., Cook, E. H., Leventhal, B. L., DiLavore, P. C., ... Rutter, M. (2000). The autism diagnostic observation schedule-generic: a standard measure of social and communication deficits associated with the spectrum of autism. Journal of Autism and Developmental Disorders, 30(3), 205-23.

Lovibond, P. F., \& Lovibond, S. H. (1995). Manual for the Depression Anxiety Stress Scales (2nd ed.). Sydney: Psychology Foundation.

Madan, C. R., Harrison, T., \& Mathewson, K. E. (2017). Noncontact measurement of emotional and physiological changes in heart rate from a webcam. Psychophysiology.

Manfredi, C., Bocchi, L., Orlandi, S., Spaccaterra, L., \& Donzelli, G. P. (2009). Highresolution cry analysis in preterm newborn infants. Medical Engineering \& Physics, 31(5), 528-532.

Mauss, I. B., \& Robinson, M. D. (2009). Measures of emotion: A review. Cognition \& Emotion, 23(2), 209-237.

McNames, J., \& Aboy, M. (2006). Reliability and accuracy of heart rate variability metrics versus ECG segment duration. Medical \& Biological Engineering \& Computing, 44(9), 747-756. http://doi.org/10.1007/s11517-006-0097-2

Oliveira-Silva, P., \& Gonçalves, Ó. F. (2011). Responding Empathically: A Question of Heart, not a Question of Skin. Applied Psychophysiology and Biofeedback, 36(3), 201. http://doi.org/10.1007/

Oller, D. K., Niyogi, P., Gray, S., Richards, J. A., Gilkerson, J., Xu, D., ... Warren, S. F. (2010). Automated vocal analysis of naturalistic recordings from children with autism, language delay, and typical development. Proceedings of the National Academy of Sciences of the United States of America, 107(30), 13354-9.

Orlandi, S., Manfredi, C., Bocchi, L., \& Scattoni, M. L. (2012). Automatic newborn cry analysis: A Non-invasive tool to help autism early diagnosis. In 2012 Annual International Conference of the IEEE Engineering in Medicine and Biology Society (pp. 2953-2956). IEEE.

R Core Team. (2014). R: A language and environment for statistical computing. Vienna, Austria: R Foundation for Statistical Computing. Retrieved from http://www.rproject.org/

Reyes-Galaviz, O. F., Cano-Ortiz, S. D., \& Reyes-García, C. A. (2008). EvolutionaryNeural System to Classify Infant Cry Units for Pathologies Identification in Recently Born Babies. In 2008 Seventh Mexican International Conference on Artificial 
Intelligence (pp. 330-335). IEEE.

Rimm-Kaufman, S. E., \& Kagan, J. (1996). The psychological significance of changes in skin temperature. Motivation and Emotion, 20(1), 63-78.

Rivard, M., Terroux, A., Parent-Boursier, C., \& Mercier, C. (2014). Determinants of stress in parents of children with autism spectrum disorders. Journal of Autism and Developmental Disorders, 44(7), 1609-20.

Robinson, M. D., \& Clore, G. L. (2002). Episodic and semantic knowledge in emotional self-report: evidence for two judgment processes. Journal of Personality and Social Psychology, 83(1), 198-215.

Rosenthal, R. (1991). Meta-Analytic Procedures for Social Research (2nd ed.). Newbury Park, CA: SAGE Publications Ltd.

Rossi, G. (1994). La rilevazione del SES in due contesti culturali italiani. AA. VV. Giornata di studio:"'Lo stile dell'interazione madre-bambino in differenti contesti culturali italiani"'. Potenza: Universitadegli studi della Basilicata.

Senese, V. P., Ruotolo, F., Ruggiero, G., \& lachini, T. (2012). The Italian Version of the Weinstein Noise Sensitivity Scale. European Journal of Psychological Assessment.

Sheinkopf, S. J., Iverson, J. M., Rinaldi, M. L., \& Lester, B. M. (2012). Atypical cry acoustics in 6-month-old infants at risk for autism spectrum disorder. Autism Research: Official Journal of the International Society for Autism Research, 5(5), 331-9.

Sheinkopf, S. J., Mundy, P., Oller, D. K., \& Steffens, M. (2000). Vocal atypicalities of preverbal autistic children. Journal of Autism and Developmental Disorders, 30(4), 345-54.

Tsai, W.-C., Tsai, J.-L., \& Lotus Shyu, Y.-I. (2008). Integrating the nurturer-trainer roles: Parental and behavior/symptom management processes for mothers of children with autism. Social Science \& Medicine (1982), 67(11), 1798-806.

Venuti, P., Caria, A., Esposito, G., De Pisapia, N., Bornstein, M. H., \& de Falco, S. (2012). Differential brain responses to cries of infants with autistic disorder and typical development: an fMRI study. Research in Developmental Disabilities, 33(6), 2255-64.

Verona, E., Patrick, C. J., Curtin, J. J., Bradley, M. M., \& Lang, P. J. (2004). Psychopathy and Physiological Response to Emotionally Evocative Sounds. Journal of Abnormal Psychology, 113(1), 99-108.

Zeskind, P. S., \& Lester, B. M. (2001). Analysis of infant crying. In L. T. Singer \& P. S. Zeskind (Eds.), Biobehavioral assessment of the infant (pp. 149-66). New York: Guilford Publications Inc.

Zhou, F., Qu, X., Jiao, J., \& Helander, M. G. (2014). Emotion Prediction from Physiological Signals: A Comparison Study Between Visual and Auditory Elicitors. Interacting with Computers, 26(3), 285-302. 